\title{
Analysis of metformin applied different tools computationals for determine all the characteristics of drug
}

\begin{abstract}
The metformin structure was analyzed using Hyperchem software to determine the structural properties for absorption process in chitosan cross linking with genipin for medical applications. The theoretical calculations were Gibbs free energy, electrostatic potential and FTIR spectroscopy. Optimized structures and their molecular electrostatic potentials were calculated using the AM1 and PM3 method, and the results were used to calculate the molecular interactions of metformin. The quantitative structure-property relationship model was also used to estimate the activity of the chemicals on the basis their molecular structures. Fourier transform infrared (FTIR) spectroscopy reveals information about the metformin properties. The molecular electrostatic potential (MESP) is a powerful tool that has provided insights into intermolecular association and molecular properties of small molecules, for example, actions of drug molecules and their analogs. The nucleophilic and electrophilic regions were calculated using the MESP. The Log $\mathrm{P}$ value ( $\mathrm{P}$ is the partition coefficient of the molecule in the water-octanol system), showed the hydrophobic character of drug.
\end{abstract}

Keywords: Metformin, Simulation, Gibbs free energy, QSAR, FTIR
Volume 2 Issue 2 - 2015

\author{
Norma Aurea Rangel Vazquez, Nancy-Liliana \\ Delgadillo Armendariz, Claudia-Lizeth Salas \\ Aguilar \\ Departamento de Metalmecanica del Instituto Tecnologico de \\ Aguascalientes, Mexico
}

\author{
Correspondence: NormaAurea RangelVazquez, \\ Departamento de Metalmecanica del Instituto Tecnologico de \\ Aguascalientes, Ave. Lopez Mateos \#180I Ote Fracc, Bona Gens, \\ CP 20256, Aguascalientes, Mexico, Tel (0I?52) 442 I9 II 840 \\ Email dranarv@gmail.com
}

Received: September 02, 2015 | Published: March 17, 2015
Abbreviations:FTIR, Fourier Transform Infrared; MESP, Molecular Electrostatic Potential; QSAR, Quantitative StructureProperty/Activity Relationships

\section{Introduction}

The major technological revolutions have shown how they can change drastically human life in all its aspects. One of the relatively new disciplines in this technological field is nanotechnology, where scientists and engineers from multiple fields discover fascinating phenomena and applications and provide us with new tools to design and exquisite novel materials where physics, chemistry, molecular biology, medicine, environment, chemical industries, pharmaceutical among others have place. ${ }^{1}$ In this context, the theoretical description and modeling of new nanodevices and various phenomena occurring in nanometric systems involve, in most of cases, concepts, calculation techniques, and theoretical approaches coming from diverse fields as physics, computational chemistry, biophysics, mathematics, medicine, optics, engineering, etc., where problems until a few years ago did not keep much to do, just being related in a fundamental way in the world nanotechnology. ${ }^{2}$

Currently, research in nanomaterials science is a vast and active field of research, both basic science and applied with a high degree of academic and technological competence. Such is the case of bionanotechnology, where the design of nanomaterials with medical applications for drug delivery, or drug transport or controlled release implants. ${ }^{1,3}$ can mimic or alter biological processes so their use in medicine could solve old problems associated with solubility, bioavailability, and cytotoxicity immune compatibility many medications traditionally used. ${ }^{4,5}$ As technology advances, the fundamental techniques of theory, modeling and simulation have been comparable to that of technological and experimental advances that have led to the development of nanoscience.

Today, thanks to the rapid development of computer technology, computer simulation has become an essential tool calculation for both experimentalists as theoretical, because through good computational model can not only be played laboratory experiments, thanks to which you can freely vary the parameters used, can prove (or distortion) theoretical models in ranges of parameters to achieve experimentally impossible for now, resolving conflicts between theoretical explanation and observation. A key role also played today displaying the results, since not only obtain numerical data that can be compared with experiments, but also get a graphic image of the process in question. ${ }^{3}$

As molecular simulation techniques are not only a useful tool for understanding and predicting the behavior of substances from a description at the molecular level, but also can predict structural, thermodynamic and transport. ${ }^{4}$ The development of empirical formulae, commonly referred to as quantitative structure-property/ activity relationships (QSAR), is an attractive approach for drug and drug delivery design. These relationships are derived from empirical correlation of physico-chemical properties of the drug or carrier molecules such as molecular descriptors, lipophilicity, electronic properties (eg. steric properties molar refractivity), quantum chemical or structural data. ${ }^{5}$ In the case of nanomedicine, computer simulation is used to create three-dimensional models, which help to better understand the behavior of these nanomaterials at sub molecular level, allowing comparisons between theory and experimental data, and even make quantitative predictions, becoming a very powerful tool to design new molecules with affinity for a given receptor. ${ }^{6-7}$

AM1 and PM3 are semi empirical method designed to analyzed different materials and determine the their structural properties like to molecular geometry, FTIR, length bond, electrostatic potential and represent a standard tool for both theoretical and experimental organic chemists. ${ }^{8-10}$ We have selected PM3 and AM1 methods to compute, because these methods are fast enough to obtain results of the calculation in minute scales using an ordinary PC. Metformin (Figure 1) used in the prevention of diabetes mellitus type 2 , is presented as an alternative solution for drugs that are primarily administered orally or 
intravenously.${ }^{11}$ Metformin works by suppressing glucose production by the liver. ${ }^{12}$

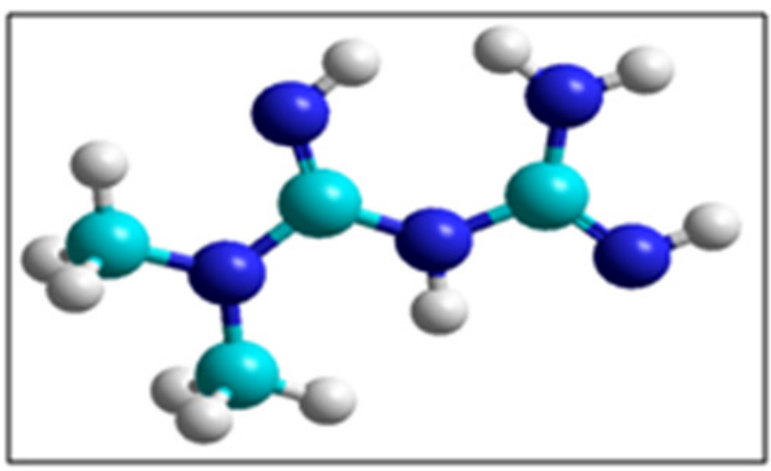

Figure I Metformine structure.

White: hydrogen; blue: carbon and purple: nitrogen atoms, respectively.

The purpose of the present work was to determine molecular geometry, FTIR, length bond and electrostatic potential of metformin using PM3 and AM1 methods, respectively.

\section{Materials and methods}

\section{Geometry optimization}

The analysis of the structure of metformin used software Hyper Chem software $8 \mathrm{v}$ using molecular mechanics and semi-empirical methods were generated. ${ }^{13}$ The geometry optimization (AM1 and PM3 methods) was calculated by means of the Polak-Ribiere algorithm with a gradient of $0.01 \mathrm{Kcal} /(\AA \mathrm{mol})$ in vacuum, 2 spin multiplicity, convergence limit of 0.0001 , and 1550 iteration limits.

\section{Electrostatic potential}

After obtaining a Gibbs free energy or geometry optimization using AM1 and PM3 methods. Hyperchem calculated the electrostatic potential maps using menu plot molecular graph option in where we realized the $3 \mathrm{D}$ representation mapped isosurface in both methods. ${ }^{13} \mathrm{In}$ fact, with these maps we can observed nucleophilic and electrophilic areas respectively, which indicate the behavior of the atomic charges.

\section{FTIR}

By vibrational and rotational option is carried out the FTIR analysis and subsequently used the vibration and rotational option of menu compute we can to appreciate the different signals from the infrared spectra, the assignment signals is realized choosing the frequency option and then is displayed the vibration signal. ${ }^{14-15}$

\section{Results and discussion}

\section{Properties}

The interaction forces between quencher and bio- molecules may include hydrophobic force, electrostatic interactions, Van der Waals interactions, hydrogen bonds, etc. ${ }^{16}$ The set of thermodynamic data obtained applied AM1 and PM3 methods are listed in Table 1. The negative value of $\Delta \mathrm{G}$ (Gibbs free energy) reflects the spontaneity of metformin.

The partition coefficient indicates the solubility of a drug in systems such as octanol/water or chloroform/water, respectively. Table 1 show the result of metformin, where the $\log P$ value calculated was 0.35 , and represents the hydrophobic properties of a substance and is considered to be a model for the absorption of molecules and widely used in oral controlled release drug delivery to obtain a desirable drug release. ${ }^{17}$ Furthermore partitioning effects apply equally to diffusion through polymer membranes. ${ }^{18}$ The analysis computational was to determine the bond length of metformin. The molecular structure along with numbering of atoms as is shown in Figure 2. The optimized structure parameter of metformin was calculated by means PM3 and AM1 and is listed in Table $2 \& 3$ respectively, these results is given in Figure 2. For this molecule, the structure is not planar and according to their observations, deformations of the ring depend on the characteristics.

Table I Properties of metformine applied simulation computational

\begin{tabular}{lll}
\hline Properties & AMI & PM3 \\
\hline$\Delta G$ (Kcal/mol) & -1745.23 & -1698.712 \\
Log P & 0.350 & 0.35 \\
\hline
\end{tabular}

Table 2 Structural parameters calculated for PVP employing PM3 and AM1 methods in metformine

\begin{tabular}{lll}
\hline Bond & AMI (Length: A) & PM3 (Length: $\mathbf{A})$ \\
\hline CI-N2 & 1.5006 & 1.5698 \\
N2-C3 & 1.5051 & 1.5414 \\
N2-C4 & 1.5229 & 1.3601 \\
C4=N5 & 1.2962 & 1.2911 \\
C4-N6 & 1.4556 & 1.3510 \\
N6-C7 & 1.4399 & 1.3462 \\
C7=N8 & 1.2875 & 1.2882 \\
C7-N9 & 1.4152 & 1.3393 \\
\hline
\end{tabular}

Table 3 Structural parameters calculated for metformine employing PM3 and AMI methods

\begin{tabular}{lll}
\hline Bond & AMI $^{(\text {Angle: }}{ }^{\circ}$ ) & PM3 (Angle: $^{\circ}$ ) \\
\hline CI-N2-C3 & 98.0911 & 107.457 \\
CI-N2-C4 & 131.127 & 136.876 \\
C3-N2-C4 & 130.782 & 125.667 \\
N2-C4=N5 & 116.079 & 122.660 \\
N2-C4-N6 & 121.001 & 117.452 \\
N5=C4-N6 & 122.920 & 119.887 \\
C4-N6-C7 & 134.110 & 149.233 \\
N6-C7=N8 & 116.159 & 124.219 \\
N6-C7-N9 & 131.422 & 126.864 \\
N9-C7=N8 & 112.419 & 118.917 \\
\hline
\end{tabular}

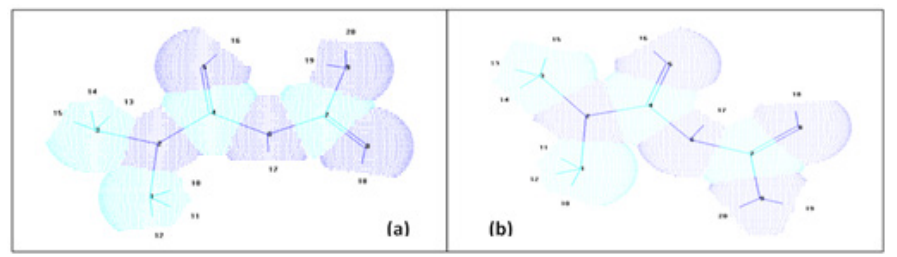

Figure 2 Metformine structure

a. AMI method

b. PM3 method.

\section{Molecular Electrostatic Potential (MESP)}

MESP is a very useful property for analyzing and predicting molecular reactive behavior. The potential has been particularly useful as an indicator of the sites or regions of a molecule that are attracted more strongly, and it has also been applied successfully to the study of interactions that involve a certain optimum relative orientation of the reactants, such as between a drug and its cellular receptor. ${ }^{19}$ The MESP values (Figure 3 ) was calculated between ( 0.056 at 0.186 $\mathrm{eV})$ and $(0.505$ at $0.171 \mathrm{eV})$ for AM1 and PM3 respectively. The electrophilic regions were appreciated at $\mathrm{NH}$ groups (atoms 5 and 9) and nucleophilic regions were localized in the $\mathrm{NH}$ (atom 17) between carbons 4 and 7. 


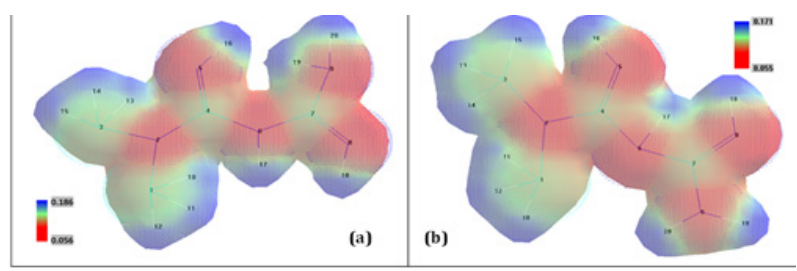

Figure 3 MESP of metformine

a. AMI method

b. PM3 method.

White: hydrogen atoms; blue: carbon atoms; purple: nitrogen atoms.

\section{FTIR}

Table 4 shows the FTIR results of metformin using AM1 and PM3 method respectively, where two characteristic bands of metformin were observed between $3615-3533$ and $3446-3420 \mathrm{~cm}^{-1}$ relative to the $\mathrm{NH}$ primary stretching vibration so of $\mathrm{NH}$ secondary stretching. ${ }^{20-23}$ Usually the frequency of this vibration is decreased in the presence of hydrogen bond. The characteristic bands at 1611, 1548-1526 and $1496 \mathrm{~cm}^{-1}$ are assigned to $\mathrm{C}-\mathrm{N}$ stretching. ${ }^{23}$ Metformin has strong absorption bands at 1840 and $1818 \mathrm{~cm}^{-1}$ which are due to $\mathrm{C}=\mathrm{N}$ stretching vibrations. It has been reported that $\mathrm{C}-\mathrm{N}$ stretching of aliphatic amine compounds is generally weak and occurs in the region $1278-1920 \mathrm{~cm}-1 .{ }^{20-21}$ The weak intensity bands in the FTIR spectra at $1611,1548-1496,1384-1092,722$ and $536 \mathrm{~cm}^{-1}$ have been assigned to C-N stretching vibrations..$^{21-24}$

Table 4 FTIR results of metformine

\begin{tabular}{|c|c|c|c|}
\hline \multicolumn{2}{|c|}{ Assignments } & \multirow{2}{*}{$\begin{array}{l}\text { AMI } \\
\text { [Frequency: } \mathrm{cm}^{-1} \text { ] } \\
3615,3533,3446,3420\end{array}$} & \multirow{2}{*}{$\begin{array}{l}\text { PM3 } \\
\text { [Frequency: cm-1] } \\
3477,3458-3445\end{array}$} \\
\hline $\mathrm{N}-\mathrm{H}$ & Stretching & & \\
\hline$C=N$ & Stretching & 1840,1818 & 1942,1930 \\
\hline $\mathrm{H}-\mathrm{N}-\mathrm{H}$ & Scissoring & 1753 & 1637 \\
\hline C-N & Stretching & $1611,1548,1526,1496$ & $|5| 2,|484-| 467$ \\
\hline $\mathrm{C}-\mathrm{N}$ & Bending & I384, I I64, I092, 536 & $1362-1298,1|2|, 618$ \\
\hline$C=N$ & Bending & 1384 & ----------- \\
\hline $\mathrm{C}-\mathrm{H}$ & Bending & $1496,1384,1359,722$ & 941 \\
\hline $\mathrm{N}-\mathrm{H}$ & Bending & 1278,532 & I209, I I88 \\
\hline
\end{tabular}

\section{Conclusion}

The results confirm that the Gibbs free energy is spontaneous in both methods. FTIR analyses showed the characteristic functional groups of the metformin. The vibrational frequency calculations revealed no imaginary frequencies, indicating that a stationary point at this level of approximation was found for the compounds. It was appreciated in the various bond lengths calculated with PM3 and AM1 levels. The MESP analysis was most important for determine the stability and molecular structure of the drug.

\section{Acknowledgments}

None.

\section{Conflicts of interest}

None.

\section{References}

1. Mendoza G, Rodriguez JL La Nanociencia y la nanotecnologia: una revolucion en curso. Perfiles Latinoamericanos. 2007;29:161-186.
2. McCurdy CW, Stechel E, Cummings P et al. Theory and Modelling in Nanoscience. Workshop Conducted by the Basic Energy Sciences and Advanced Scientific Computing Advisory Committees to the Office of Science, Department of Energy, USA. 2002

3. Hofer TS From macromolecules to electrons-grand challenges in theoretical and computational chemistry. Front Chem. 2013;1:6.

4. Leach A Molecular Modelling: Principles and Applications. (2nd edn), Prentice Hall Ltd., New Jersey, USA. 2001

5. Haddish-Berhane N, Rickus JL, Haghighi K The role of multiscale computational approaches for rational design of conventional and nanoparticle oral drug delivery systems. Int $J$ Nanomedicine. 2007;2(3):315-331.

6. Gago BF Metodos computacionales de modelado molecular y diseño de fármacos. An Real Acad Nac Farm. 1993;253-327.

7. Buch I, Giorgino T, Fabritiis G Complete reconstruction of an enzymeinhibitor binding process by molecular dynamics simulations. Proc Nat Acad Sci USA. 2011;108(25):10184-10189.

8. Hwang W, Hwang Y, Lee S, Lee D Rule-based multi-scale simulation for drug effect pathway analysis. BMC Med Inform Decis Mak. 2013;13(Suppl 1):1-9.

9. Kalninsh KK Joint experimental and theoretical study of the poly (styryl sodium) and poly ( $\alpha$-methyl styryl sodium) polymerization depolymerization. P hys Chemi Chem Phys. 2001;3(20):4542-4546.

10. Klein E, Matis M, Lukes V, Cibulkova Z The applicability of AM1 and PM3 semi-empirical methods for the study of $\mathrm{NeH}$ bond dissociation enthalpies and ionization potentials of amine type antioxidants. Polym Degrad Stabil. 2006;91(2):262-270.

11. Mertins O, Dimova $\mathrm{R}$ Binding of Chitosan to Phospholipid Vesicles Studied with Isothermal Titration Calorimetry. Langmuir 2011;27(9):5506-5515.

12. El Messaoudi S, Rongen GA, De-Boer RA et al. The cardioprotective effects of metformin. Curr Opin Lipidol. 2011;22(6):445-453.

13. Ravindran CA, Yoke JC, Sheau TC et al. In vitro studies and evaluation of metformin marketed tablets-Malaysia. J App Pharmaceut Sci. 2011;1(5):214-217.

14. Kumirska J, Czerwicka M, Kaczyn ski Z et al. Application of Spectroscopic Methods for Structural Analysis of Chitin and Chitosan. Mar Drugs. 2010;8(5):1567-1636.

15. Rangel-Vázquez NA Computational Chemistry Applied in the Analyses of Chitosan/Polyvinylpyrrolidone/Mimosa Tenuiflore. (1st edn), Science Publishing Group, Hong Kong, pp. 2013;10-15.

16. Ahmad TA, Jahan R, Abdul QM et al. Spectroscopic Studies of the Interaction between Metformin Hydrochloride and Bovine Serum. Dhaka Univ J Pharm Sci. 2012;11(1):45-49.

17. Hardinnawirda K, Sitirabiatull I Effect of rice husks as filler in polymer matrix composites.JMech Eng Sci. 2012;2:181-186.

18. Narasimharao R, Reddy MA, Reddy NS Design and Evaluation of Metformin Hydrochloride Extended Release Tablets by Direct Compression. Int J ResPharmaceut Biomed Sci. 201;2(3):1118-1133.

19. Politzer P, Laurence PR, Jayasuriya K Molecular electrostatic potentials: an effective tool for the elucidation of biochemical phenomena. Environ Health Perspect. 1985;61:191-202.

20. Gary CD Analytical Chemistry. (6th edn), John Wiley and Sons, Hoboken, USA. 2003

21. www. depa.fquim.unam.mx/amyd/archivero/Tabla de IR 20785

22. Sheela NR, Muthu S, Krishnan SS FTIR, FT Raman and UV-Visible Spectroscopic Analysis on Metformin Hydrochloride. Asian J Chem. 2010;22(7):5049-5056. 
23. Fahim F, Naseer A, Ahmed S, Tufail S, Bhanger MI A Green Approach for the Determination of Selected Anti-Diabetic Drugs in Pharmaceutical Formulation by Transmission FTIR Spectroscopy. J Braz Chem Soc. 2014;25(11):2032-2038.
24. Renganayaki V, Srinivasan S HF, DFT Computations and Spectroscopic Study of the Vibrational and Thermodynamic Properties of Metformin. Int J Pharm Tech Research. 2011;3(3):1350-1358. 